\title{
ORGANIZACIÓN Y DESARROLLO DE LA ESFERA DE LOS SERVICIOS EN CUBA
}

\author{
POR \\ ISABEL MARÍA VALDIVIA FERNÁNDEZ
}

Con el triunfo de la Revolución cubana, el primero de enero de 1959, se inicia un proceso de transformaciones radicales de las estructuras socioeconómicas del país. La propiedad social se extedió a todos los sectores de la economía, exceptuando una pequeña parte del transporte en manos privadas y de la tierra perteneciente a agricultores individuales, área que se integra progresivamente, a través del proceso de cooperativización fundamentado en la más estricta voluntariedad.

$\mathrm{Al}$ comenzar la construcción del socialismo, bajo el bloqueo económico de los Estados Unidos, el impulso inicial en la edificación socialista se dirigió fundamentalmente hacia el desarrollo social, en correspondencia con las necesidades insatisfechas del pueblo. Una parte importante de los recursos se dedicó a esos fines, particularmente a la educación y a la salud pública. En la esfera económica, el esfuerzo principal se orientó a la reestructuración de la agricultura, de la producción azucarera y a la creación y desarrollo de la industria pesquera, a fin de incrementar los fondos exportables tradicionales y elevar la disponibilidad de productos alimenticios destinados al consumo interno. A partir de 1975 se impulsa el crecimiento de las ramas fundamentales de la industria, las construcciones, el transporte, las comunicaciones y otros sectores, desarrollando en todos estos

Estudios Geográficos

Tomo LVII, n. ${ }^{\circ} 223$, abril-junio 1996 
años de manera significativa la base económica del país, lo que ha permitido lograr avances importantes.

La primera etapa de la acción revolucionaria correspondió al proceso de redistribución de bienes y servicios en función de resolver los aspectos más injustos de las contradicciones sociales. Así una parte importante de las inversiones se desvió hacia las zonas rurales en forma de servicios médicos, escuelas y carreteras.

El desarrollo alcanzado en la salud pública y en la educación ha constituido un factor de gran importancia. La dotación de una red de hospitales y policlínicos en todo el país y de servicios educacionales, generales y especializados, ha significado la creación en todas las provincias y municipios de una fuerza de trabajo calificada, lo que influyó en la reestructuración de los asentamientos, en la disminución de las diferencias territoriales y en la desconcentración de estos servicios, que sólo existían en La Habana.

Los servicios de salud en Cuba se caracterizan, en primer lugar, porque la salud constituye una responsabilidad del Estado, de ahí que se estructure como un Sistema Nacional (véase fig. 1); por otra parte los servicios médicos son accesibles y gratuitos para toda la población $\mathrm{y}$, por último, tienen un carácter integral de prevención, curación y promoción de salud. El 23 de enero de 1960 se promulgó la Ley 723, creándose el Servicio Médico Rural. Se inició de forma acelerada la construcción de una red de hospitales y postas médicas que permitían garantizar la atención médica a toda la población rural. De esta forma, la asistencia médica y hospitalaria, gratuita para todos los ciudadanos, se llevó hasta los más apartados rincones de la nación.

A pesar del masivo éxodo de profesionales de la salud, el programa de preparación del personal médico y paramédico llevado a cabo, logró que se incrementara el número de médicos y personal calificado del sector, así como se priorizó la atención a las madres y niños, lo cual influyó en la vertiginosa disminución de la tasa de mortalidad infantil, 9,4 por cada 1.000 en 1995 (Granma, 1996). Los índices que miden hoy el estado de salud de la población constituyen uno de los más importantes logros del estado cubano, aproximándose algunos de ellos a los países desarrollados; la esperanza de vida al nacer, en 


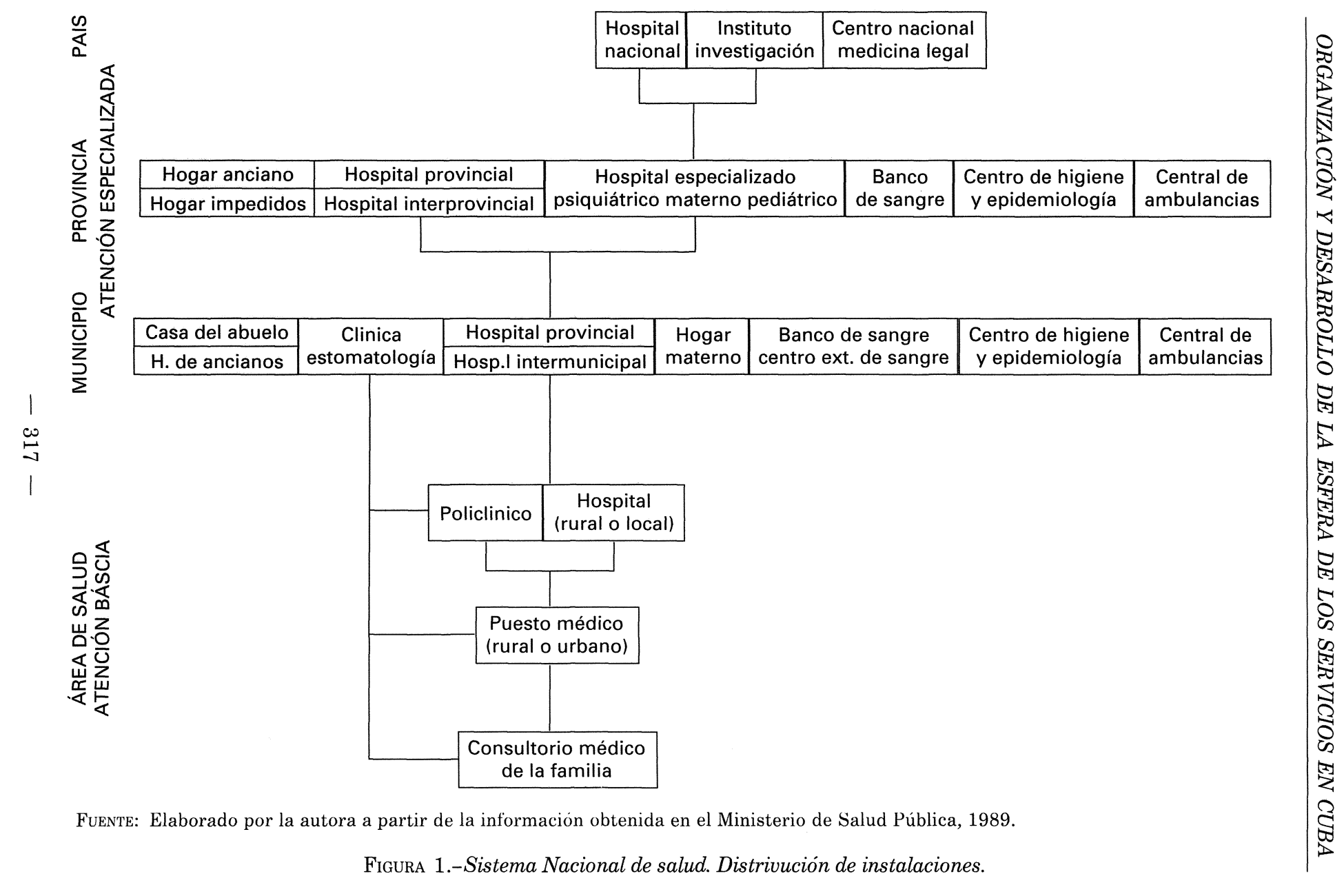


estos momentos, es como promedio de setenta y cinco años (Ministerio de Salud Pública, 1993).

El número de médicos por habitantes fue de 1/258 y el de estomatólogos de 1/1.436 en 1993 (Ministerio de Salud Pública, 1993). El Plan del Médico de la Familia cuenta ya con más de 15.000 médicos y enfermeros que prestan servicio a lo largo del país, no sólo en las zonas de residencia de la población, sino también en escuela, círculos infantiles, fábricas y centros de trabajo en general; éstos tienen como función fundamental la prevención y curación de enfermedades, además de la educación higiénico-sanitaria de la población que atienden. Se prevé que para el 2000 esta cifra alcance los 20.000 médicos y enfermeros que atenderán a toda la población (Ministerio de Salud Pública, 1993). En Cuba, la satisfacción de la demanda en hospitales para el año 2000 se calcula en 8 camas/1.000 habitantes. Los policlínicos presentan características semejantes al servicio hospitalario, calculándose para ese mismo año un índice de satisfacción de 8,5 consultas/habitante/año (IPF, 1992).

En el camo de la educación se han logrado avances importantes. La enseñanza se extendió a toda la población desde el primer año de la Revolución y en 1961 fue nacionalizada, dando inicio a profundos cambios, que hicieron posible en ese mismo año eliminar el analfabetismo heredado y que afectaba a casi un millón de personas. El sistema de educación en Cuba se basa en tres principios fundamentales, la escuela estatal, gratuidad en los servicios y educación integral. Con el objetivo de llevar la enseñanza hasta los más apartados rincones del país, se construyó una red de instalaciones educacionales que lo garantizan paras los diferentes niveles de enseñanza, la cual se encuentra distribuida tanto en las zonas urbanas como rurales (véase cuadro I).

Por otra parte, la creación de las escuelas en el campo con carácter interno, además de cumplir con su objetivo formador a partir de la vinculación del estudio y el trabajo, conllevó, también, a cambios en las formas de organización social de la producción, que influyen en la concepción de la contradicción ciudad-campo, ya que no sólo se vincula la actividad educacional a las áreas urbanas, sino que posibilita el desarrollo de nuevos territorios subutilizados y permite la eli- 
Cuadro I

INSTALACIONES Y SUS CARACTERÍSTICAS

\begin{tabular}{|c|c|c|}
\hline Niveles de Enseñanza & Tipo de Instalación & Localización \\
\hline Pre-escolar & $\begin{array}{l}\text { Círculo infantil } \\
\text { o Escuela Primaria }\end{array}$ & Asentamientos urbanos \\
\hline & $\begin{array}{l}\text { Escuela Primaria Multígrada } \\
\text { Escuela Primaria Graduada }\end{array}$ & $\begin{array}{l}\text { Área rural } \\
\text { Área rural y urbana }\end{array}$ \\
\hline Enseñanza Primaria & $\begin{array}{l}\text { Seminternado de Primaria } \\
\text { Internados de Primaria }\end{array}$ & $\begin{array}{l}\text { Asentamientos urbanos } \\
\text { Área rural y urbana }\end{array}$ \\
\hline \multicolumn{3}{|l|}{ Enseñanza Media: } \\
\hline Externa & $\begin{array}{l}\text { Escuelas Secundarias Básicas } \\
\text { y Preuniversitarios urbanos }\end{array}$ & Asentamientos urbanos \\
\hline Interna & $\begin{array}{l}\text { Escuela de enseñanza media } \\
\text { básica y superior }\end{array}$ & Área rural \\
\hline Especializada & $\begin{array}{l}\text { Escuelas Vocacionales, de Arte, } \\
\text { Deporte, Militar, etc. }\end{array}$ & Área rural y urbana \\
\hline Técnica y profesional & $\begin{array}{l}\text { Escuelas e Institutos } \\
\text { Politécnicos }\end{array}$ & Áreas rural y urbana \\
\hline Enseñanza especializada & $\begin{array}{l}\text { Escuelas especiales para } \\
\text { alumnos con dificultades físicas } \\
\text { débiles visuales }\end{array}$ & Asentamientos urbanos \\
\hline
\end{tabular}

FuEnte: Franco, X., y Valdivia, I., 1992.

minación paulatina de los desniveles culturales entre los territorios. El número de matriculados cada año se incrementa, y a pesar de la situación económica por la que atraviesa el país, no ha dejado de inaugurarse ningún curso escolar, abriendo sus puertas todas las escuelas los primeros días del mes de septiembre.

Otro logro a destacar en el desarrollo de la educación en Cuba lo constituye la educación diferenciada especializada para niños y jóvenes con problemas o limitaciones, ya sean físicas, psíquicas, visuales,

$$
\text { - } 319-
$$


auditivas, etc., los cuales estudian en escuelas especiales atendidos por un personal calificado, preparado para estos fines.

En cuanto a la vivienda, además del crecimiento absoluto que representa más de un millón de viviendas, la calidad del fondo ha tenido una evolución significativa, habiendo disminuido las viviendas en mal estado de un $47 \%$ a un $28 \%$, e incrementándose las buenas y regulares de un $53 \%$ a un $72 \%$ (IPF, 1992). En 1960 se dictó la Ley de Reforma Urbana que hizo propietario a los inquilinos mediante amortización por las mismas mensualidades y durante un número de años relacionados con la fecha de construcción de la vivienda. A partir de este momento, para las viviendas que construye el Estado, se establece un límite máximo de pago mensual de un $10 \%$ del ingreso familiar. Se comenzaron los programas masivos de construcción de viviendas urbanas y rurales y se construyeron más de 320 nuevas comunidades en zonas agrícolas o agroindustriales, lo cual ha contribuido a elevar el nivel de vida de la población, atenuar las diferencias existentes entre la ciudad y el campo y lograr un desarrollo equilibrado de los diferentes territorios, frenando el crecimiento hipertrofiado de la capital. En diciembre de 1984, se aprobó la Ley General de la Vivienda, que culmina los propósitos y aspiraciones de las primeras leyes sobre la vivienda.

Cuba dispone en estos momentos de un fondo de viviendas de poco más de 2,7 millones. Se calcula que en la década de los noventa se desprecien 1,1 millón de viviendas, por lo que el fondo útil al año 2000 quedaría en 1,6 millones (IPF, 1992). Por otra parte, la demanda para finales de siglo se calcula en unos 3,5 millones de viviendas (una población de 11,6 millones, a 3,3 habitantes por familia), por lo que la diferencia se eleva a 2,0 millones de unidades (IPF, 1992). Pretender resolver este problema, en breve plazo, está realmente fuera del alcance de las posibilidades del país, dada la situación existente.

Entre las características de la cultura cubana tenemos su homogeneidad, a pesar de sus diversas raíces, esta uniformidad y consolidación viene dada, principalmente por la ausencia de barreras topográficas y de otra índole; otra característica es el internacionalismo, lo cual tiene su origen en la pluralidad de sus orígenes étnicos. A partir de 1959 comienza un trabajo dirigido a enriquecer la vida espi- 
ritual del hombre y a partir de la Campaña de Alfabetización se establecieron premisas indispensables para una nueva dimensión cultural. Una amplia red de instituciones culturales de todo tipo se construyó en el país, posibilitando que a lo largo y ancho de éste, la población disponga de bibliotecas, museos, casas de cultura, cines, etc., lo cual constituye la célula básica del trabajo cultural en la comunidad. Actualmente el patrimonio cultural atesora una gran riqueza, tanto en piezas museables, libros, documentos, producción literaria, plástica, musical, entre otras, al alcance de todos.

El derecho de todo el pueblo al deporte se ha garantizado y se ha eliminado el mercantilismo en su práctica y desarrollo. Como un reflejo más de la situación económica y social existente en Cuba antes de 1959, el desarrollo de la cultura física y el deporte era muy limitados; las instalaciones existentes tenían un carácter privado y se concentraban principalmente en la capital del país. Esta situación determinó la necesidad de crear programas y métodos que estimularan a la población a la práctica de la educación física y el deporte. Se construyeron un gran número de instalaciones por todo el país, así como escuelas para profesores de educación física y de formación de deportistas. Una constante en el desarrollo de esta rama ha sido la búsqueda de la masividad en su práctica, de ahí el gran número de instalaciones construidas en los diferentes asentamientos, asegurando el acceso a la participación deportiva de la población urbana y rural.

La organización territorial de esta rama se concibe de forma escalonada, de forma tal que en los asentamientos más pequeños encontramos instalaciones que permiten la práctica de los deportes más populares y en la medida en que aumenta la categoría de los mismos, las opciones crecen, contando con más disciplinas. Este criterio de localización varía cuando estas instalaciones están vinculadas al deporte como espectáculo, adquiriendo importancia extraurbana, lo que aumenta sus isocronas de accesibilidad.

En general, tanto el deporte como la cultura física, han mantenido un desarrollo ascendente y sistemático, lográndose un salto cuantitativo y cualitativo en cuanto al número de deportistas de alto rendimiento, cantidad de instalaciones, títulos obtenidos internacionalmente, etc. Otro logro en esta rama es la incorporación a la prác- 
tica de deportes de los discapacitados, que incluyen en sus programas además de las prácticas, la celebración de competencias a diferentes niveles, incluso internacional, donde se han obtenido magníficos resultados. Como principal problema de esta rama se puede plantear, a pesar de los esfuerzos realizados y programas desarrollados, que aún es bajo el nivel de utilización de las instalaciones existentes por parte de la población. Se ha impulsado además la implantación y mejoramiento cualitativo de la recreación y de otros servicios sociales, puestos en función de las necesidades del desarrollo integral del hombre.

El cambio de la configuración urbana de numeros pueblos y ciudades de Cuba, caracterizado por la multiplicación de las instalaciones de tipo económico y social, la edificación de nuevas comunidades rurales, expresan los esfuerzos encaminados al desarrollo armónico e integral del país, y a la disminución de los factores que en el pasado propiciaban la emigración del campo a la ciudad, a la vez que permite cierto grado de concentración poblacional, acorde con los objetivos de carácter económico y social que se ha trazado la Revolución.

El desarrollo de las nuevas inversiones en este campo se ha realizado teniendo en cuenta una acertada distribución territorial de las fuerzas productivas, acercando los centros de producción a las fuentes de materias primas, de fuerza de trabajo, a las vías de comunicaciones. Ello conduce al acercamiento paulatino de los niveles de desarrollo de los diferentes territorios.

Para la organización y desarrollo de la esfera de los servicios después de la Revolución, ha sido importante contar con los resultados de las investigaciones que sobre el Sistema de Asentamientos Humanos ha desarrollado, desde los primeros años de la década de los setenta, el Instituto de Planificación Física (IPF); lo que ha contribuido al perfeccionamiento de la estructura territorial de los sistemas de servicios sociales y del hábitat y a una mejor planificación de los mismos. En Cuba se ha estudiado un modelo de urbanización basado en la estructuración del Sistema de Asentamientos Poblacionales (SAP) dentro de una unidad geográfica, el subsistema, donde se producen relaciones entre los asentamientos de diferente categoría y el territorio (véase fig. 2). En el subsistema se establecen las relaciones funcionales de producción y servicios más significativas entre los lugares, permitien-

$$
-322-
$$




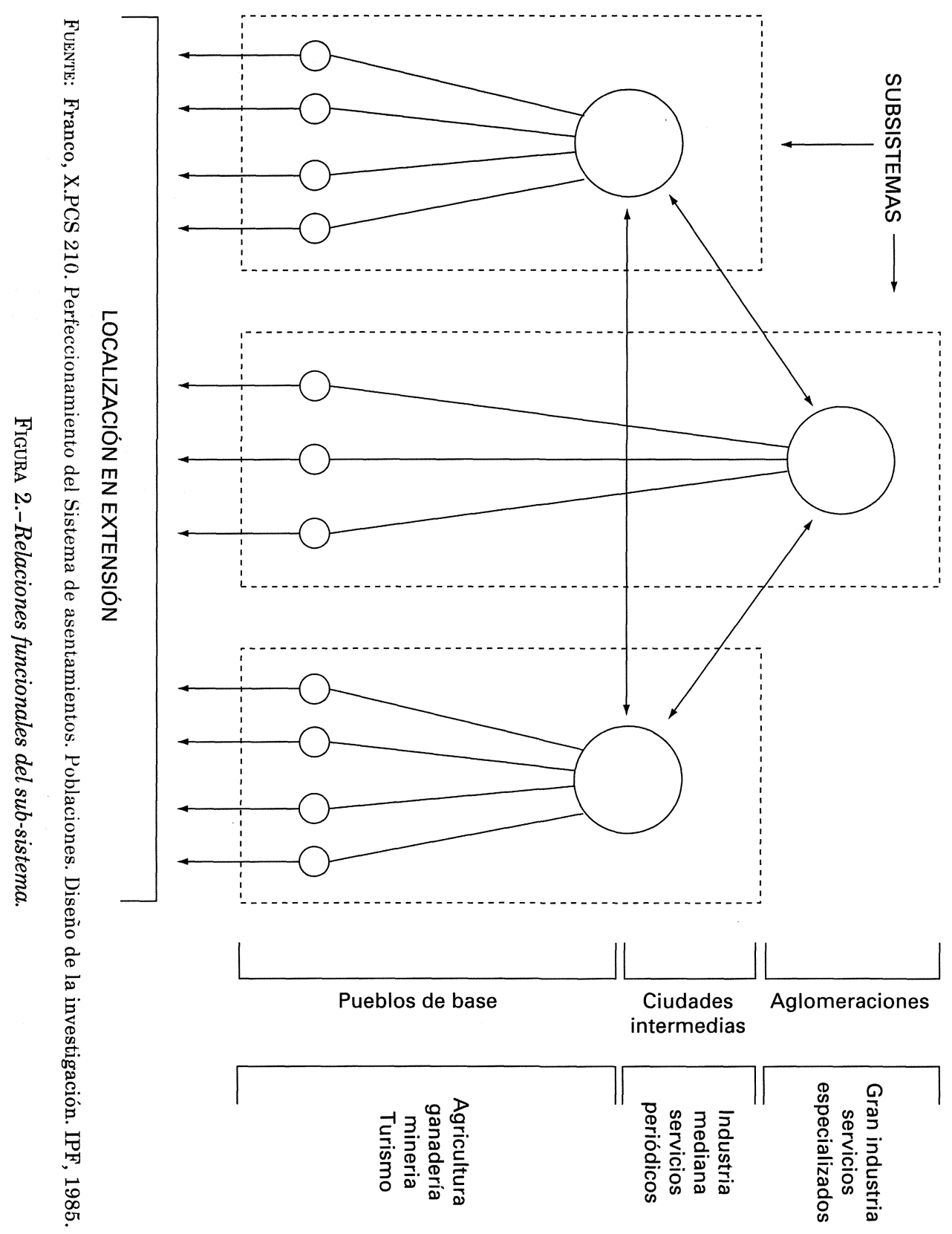

Localización puntual

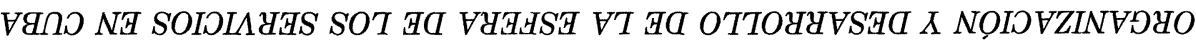


do la accesibilidad a los servicios intermedios en isocronas de tiempo lógico, asegurando así la elevación constante del nivel de vida y la paulatina eliminación de las diferencias entre la ciudad y el campo.

La base material que garantiza la prestación de los servicios sociales tiene una serie de características funcionales definidas por el tipo de usuario, la frecuencia de uso, el servicio que brinda, etc., que hacen que su nivel de aparición territorial sea diferenciado; hasta ahora, el análisis de los servicios e instalaciones se había estado haciendo a través de una serie de normas con un enfoque parcial o fragmentario y que no contemplaban las características propias de cada escala territorial. Teniendo en cuenta lo anterior, en Cuba fue aprobado en 1982 el Programa de Normas sobre la Infraestructura Social y la Vivienda (PNISV) que tiene como objetivo elaborar un sistema de normas para la localización y dimensionamiento de las instalaciones de la infraestructura social y la vivienda.

Las normas relativas a la Infraestructura Social están subdividida en cuatro niveles normalizativos que van desde el programa por tipo de subsistemas, pasando por el programa por tipo de asentamientos, los requerimientos para la ubicación de las instalaciones en el ámbito urbano, hasta el programa y dimensionamiento de las instalaciones. En estas normas se establecen los tipos de asentamientos humanos y de subsistemas a utilizar, se define la nomenclatura de las instalaciones de servicio correspondiente a cada tipo, en función de su frecuencia de uso: diaria, periódica y esporádica. A su vez se define para cada tipo de servicio los factores de localización siguiente: población servida, tamaño de los módulos, frecuencia de uso, accesibilidad o indicadores económicos funcionales. Las normas son de obligatoria aplicación en elaboración de los esquemas de desarrollo y distribución de servicios, tanto nacional como provincial, así como en los esquemas de desarrollo de las ciudades, que comenzaron a elaborarse en Cuba en 1980.

Estos esquemas están basados en la necesidad de estructurar el sistema de los servicios sociales en el territorio, con vistas a alcanzar una distribución tal que posibilite conjugar los intereses ramales con la distribución territorial de la población, un aprovechamiento más racional de los fondos y una estructuración más efectiva de las inversiones, todo ello en aras de lograr una óptima organización y desarrollo

$$
-324-
$$


de la esfera de los servicios en el país y con ello condiciones más homogéneas de vida en los distintos niveles del sistema de asentamientos.

A lo largo de estos últimos diez años, se han llevado a cabo nuestras investigaciones, cuyos resultados han evidenciado la necesidad de revisar este instrumental normativo, dándole un enfoque más realista, flexible y creativo con el fin de acercamiento de accesibilidad a los servicios de toda la población residente en los diferentes niveles del SAP, racionalizando las instalaciones que se demandan en función de horarios, régimen de explotación más intenso, uso múltiple, etc., evitando así la subutilización de las mismas. Por otra parte, esta flexibilidad, ha permitido lograr una mayor participación comunitaria en la búsqueda de soluciones a los problemas concretos que se presentan en cada asentamiento.

\section{BIBLIOGRAFÍA}

Angulo, J.; Morales, J., y otros (1991): La cultura cubana, La Habana.

Franco, X., e Valdivia, I. M. (1992): Geografia de la esfera de los servicios, Universidad de La Habana (inédito).

HERRERA, A., y otros (1996): Geografía, población y ordenamiento del medio rural, Universidad de Toulouse-Le Mirail, Francia (en prensa).

InSTITUTO DE PLANIFICACION FISICA (1992): PCS. 210, Perfeccionamiento del sistema de asentamientos poblacionales, La Habana (inédito).

Ministerio de CUltuRa (1992): Estadisticas de Archivo, La Habana.

MiNiSTERIO DE EDUCACIÓN (1993): Estadísticas de Archivo.

MiNisterio de SALUd PÚBLICA (1993): Estadisticas de Archivo.

REPÚBLICA DE CUBA (1992): Gaceta oficial julio-agosto.

- (1992): Gaceta oficial septiembre-octubre.

RESUMEN. La esfera de los servicios constituye una parte importante de la economía nacional. Un pobre desarrollo de esta esfera frena el progreso de la sociedad e influye negativamente en su auge económico. El presente artículo se propone dar una idea de las características de la organización y desarrollo de esta esfera en Cuba. Dado el amplio carácter de la esfera, por la variedad y número de ramas que contiene, así como su desarrollo en el tiempo, el mismo no presenta un análisis detallado de cada una de las ramas que la conforman ni su desarrollo histórico, sino más bien destacar algunos elementos relevantes por el papel que han jugado y juegan en el mejoramiento de las condiciones de vida de la población en general.

Palabras Claves: Esfera de los servicios. Organización territorial. Satisfacción de demandas. Sistema de asentamientos poblacionales. 